\title{
CAPACIDADE DE DUAS FONTES DE SELÊNIO EM REDUZIR HEPATOTOXICIDEZ CAUSADA PELA INGESTÃO DE AFLATOXINAS
}

\author{
ANTONIO SAMPAIO BAPTISTA* \\ ADIBE LUIZ ABDALLA* \\ DEISE DA SILVA PIRES* \\ ANDRÉIA CABRINI ZAMPRONIO* \\ CAROLINE ROSSIM LOUVANDINI* \\ VÍRGILIO FRANCO DO NASCIMENTO FILHO** \\ MARIAANTONIA CALORI-DOMINGUES ${ }^{* * *}$ \\ EDUARDO MICOTI DA GLÓRIA*** \\ MÁRIO ROBERTO VIZIOLI**** \\ JORGE HORII***
}

Dezoito ratos wistar foram utilizados para investigar os efeitos de selenito de sódio $\left(\mathrm{Na}_{2} \mathrm{SeO}_{4}\right)$ e de selênio, proveniente de leveduras ( $\mathrm{SeL}$ ) sobre os danos induzidos por aflatoxinas. Os animais foram distribuídos em seis grupos de tratamento com três repetições cada: grupo 1 = controle; grupo 2 = $480 \mu \mathrm{g} \mathrm{kg}^{-1}$ de aflatoxinas (AF); grupo $3=0,5 \mathrm{mg}$ de $\mathrm{Na}_{2} \mathrm{SeO}_{4}$ por kg de dieta; grupo $4=0,5 \mathrm{mg}$ de $\mathrm{SeL}$ por $\mathrm{kg}$ de dieta; grupo $5=\mathrm{AF}+0,5 \mathrm{mg}$ de $\mathrm{Na}_{2} \mathrm{SeO}_{4}$ por $\mathrm{kg}$ de dieta e grupo $6=\mathrm{AF}+0,5 \mathrm{mg}$ de SeL por $\mathrm{kg}$ de dieta. Aos 28 dias de ensaio os animais foram sacrificados para exame histopatológico. Os animais do grupo AF evidenciaram poucos sinais de hepatotoxicidade. Nos grupos $\mathrm{AF}+\mathrm{Na}_{2} \mathrm{SeO}_{4}$ e $\mathrm{AF}+$ $\mathrm{SeL}$, os animais apresentaram fígados muito bem preservados. Os grupos que receberam $\mathrm{Na}_{2} \mathrm{SeO}_{4} \mathrm{e}$ SeL revelaram algumas alterações. Conclui-se que o selenito de sódio e SeL evidenciaram potencial para diminuir os danos provocados por aflatoxinas.

* Laboratório de Nutrição Animal, Centro de Energia Nuclear na Agricultura (CENA) Universidade de São Paulo (USP), Piracicaba, SP (e-mail: asbaptis@ciagri.usp.br).

** Laboratório de Instrumentação Nuclear, CENA, USP, Piracicaba, SP.

*** Departamento de Agroindústria, Alimentos e Nutrição, Escola Superior de Agricultura Luiz de Queiroz (ESALQ/USP), Piracicaba, SP.

**** Departamento de Diagnóstico Oral, Faculdade de Odontologia de Piracicaba (FOP), Universidade de Campinas (UNICAMP), Piracicaba, SP. 


\section{INTRODUÇÃO}

A cada dia aumenta a preocupação com a qualidade de vida das pessoas. A produção e o aproveitamento de alimentos saudáveis têm participação importante para o bem-estar e à saúde humana. Entretanto, é corriqueira a presença de substâncias nocivas em produtos destinados à alimentação. Merece atenção a ocorrência de micotoxinas, que são moléculas altamente tóxicas e que podem oferecer sérios riscos à sanidade de humanos e de animais. Entre as micotoxinas, as aflatoxinas são as mais conhecidas por serem as mais tóxicas. As aflatoxinas são produzidas por algumas linhagens Aspergillus flavus, $A$. parasiticus e $A$. nomius e quando ingeridas podem provocar sérios prejuízos à saúde humana ou de animais (HEATHCOTE e HIBBERT, 1978; ELLIS, SMITH e SIMPSON 1991).

Bioquimicamente, as aflatoxinas podem afetar os metabolismos de energia, de carboidratos, de lipídios, dos ácidos nucléicos e das proteínas. Os efeitos biológicos envolvem carcinogenicidade, mutagenicidade, teratogenicidade, hepatotoxicidade e aflatoxicoses (ELLIS, SMITH e SIMPSON, 1991).

O impacto econômico resultante da contaminação por aflatoxina ocorre em todos os níveis da produção vegetal e animal envolvidos, comercialização da produção e utilização dos produtos (KUBENA et al., 1990). A facilidade e freqüência com que as aflatoxinas contaminam os produtos agrícolas, bem como a exposição de animais a esses compostos químicos via dieta contaminada, podem ser as principais diferenças entre o lucro e o prejuízo em muitas atividades da agroindústria (HAMILTON, 1984).

Entre os possíveis agentes que podem ser utilizados para a minimizar os danos promovidos pelas aflatoxinas encontram-se os agentes antioxidantes, dentre os quais pode-se destacar o selênio (LEl et al., 1990). Além de fornecer inúmeros benefícios à saúde animal e humana, o selênio (Se) tem sido apontado como agente promissor para a inibição de danos promovidos por micotoxinas, em especial, pelas aflatoxinas (LEl et al., 1990; SHI, HEW e ONG 1995). A função do selênio está ligada à enzima glutationa peroxidase (GPS-Px), uma oxirredutase que evita a formação de lipoperóxidos tóxicos. A descoberta da sua participação junto a GSP-Px foi a chave para o entendimento da sua importância em nutrição e saúde (ROTRUCK et al., 1973).

Em níveis não-tóxicos, o selênio tanto na forma inorgânica como na forma orgânica tem demonstrado eficiência na inibição da citotoxicidade induzida por aflatoxina $B_{1}$. A forma orgânica é menos tóxica do que a inorgânica, entretanto, a forma inorgânica é mais eficaz para inibir a morte celular induzida por aflatoxinas (SHI, HEW e ONG 1995).

Os sérios riscos à saúde pública oferecidos pelas aflatoxinas e a possibilidade promissora de se minimizar aflatoxicoses com a utilização de Se tornam relevante o desenvolvimento de pesquisas esclarecedoras nesse sentido. Desta forma, o propósito deste trabalho foi investigar os efeitos promovidos por aflatoxinas em presença de dietas suplementadas com Se.

\section{MATERIAL E MÉTODOS}

Dezoito ratos albinos (Rattus norvegicus) da linhagem Wistar, com 21 a 25 dias de idade e pesando aproximadamente 65 gramas cada, foram distribuídos em gaiolas individuais e separados inteiramente ao acaso em seis grupos de tratamentos (com 3 repetições cada) para avaliar os efeitos promovidos por aflatoxinas na presença de dietas suplementadas com selênio. Os tratamentos foram:

- grupo 1 = controle livre de aflatoxinas (AF), níveis não-detectáveis (ND) $\leq 0,5 \mu \mathrm{g} \mathrm{kg}^{-1}$;

- $\quad$ grupo $2=480 \mu \mathrm{g} \mathrm{kg}^{-1}$ de AF - média (M) $\left(255 \mu \mathrm{g} \mathrm{kg}^{-1}\right.$ por 10 dias e $600 \mu \mathrm{g} \mathrm{kg}^{-1}$ por 18 dias);

- $\quad$ grupo $3=\mathrm{AF}(\mathrm{M})+0,5 \mathrm{mg}$ de $\mathrm{Na}_{2} \mathrm{SeO}_{4}$ por kg de dieta; 
- $\quad$ grupo $4=\mathrm{AF}(\mathrm{M})+0,5 \mathrm{mg}$ de Sel por kg de dieta;

- $\quad$ grupo $5=0,5 \mathrm{mg}$ de $\mathrm{Na}_{2} \mathrm{SeO}_{4}$ por kg de dieta; $\mathrm{e}$

- $\quad$ grupo $6=0,5 \mathrm{mg}$ de SeL por $\mathrm{kg}$ de dieta.

Os animais foram alimentados durante 28 dias com dieta a base de proteína, carboidratos, lipídios, fibras, mistura mineral e mistura vitamínica, dieta AIN-93G, descrita por REEVES, NIELSEN e FAHEY (1993). Os animais receberam água "ad libitum" e 15 gramas de dieta (cada animal) diariamente. Efetuou-se a contaminação das dietas pela adição de amendoim naturalmente contaminado por aflatoxinas. Foi utilizado $\mathrm{Na}_{2} \mathrm{SeO}_{4}$ obtido no comércio local de Piracicaba e SeL do Departamento de Agroindústria e Nutrição, Escola Superior de Agricultura Luiz de Queiroz, Universidade São Paulo. Todas as dietas foram estocadas em câmara fria até seu o fornecimento aos animais.

A determinação de aflatoxinas foi realizada por cromatografia em camada delgada, conforme metodologia descrita por SOARES e RODRIGUES-AMAYA (1989), com adaptações como a quantificação mediante cromatografia bi-direcional em que o limite de detecção para as quatro aflatoxinas é de $0,5 \mu \mathrm{g} \mathrm{kg}^{-1}$.

Após 28 dias de ensaio, os animais foram anestesiados em câmara saturada com halotano. Em seguida, tiveram suas cavidades abdominais abertas e seus órgãos internos (fígado, coração, rins e baço) extraídos e pesados. Os fígados foram fixados em solução de formol $10 \%$ até o preparo para a histologia. Logo após o sacrifício, realizou-se a coleta de sangue dos animais.

As lâminas histológicas, preparadas conforme as técnicas histológicas descritas no Compêndio básico de técnicas histológicas (PACHECO, 1981), foram coradas com hematoxilina e eosina (HE). Após o preparo, as lâminas foram observadas ao microscópio de luz com aumento de 16 x 1,25 x $10(200 x)$.

Os tecidos hepáticos dos animais que receberam dieta livre de aflatoxinas foram adotados como padrão de referência para isenção de toxicidade.

Os resultados obtidos foram submetidos à análise da variância e ao Teste de Comparação de Médias de Tukey ao nível de significância de 5\%, usando-se o programa SAS (HERZBERG, 1990).

\section{RESULTADOS E DISCUSSÃO}

\subsection{APROVEITAMENTO ALIMENTAR E PESOS DE ÓRGÃO INTERNOS}

As médias de consumo de alimento, de ganho de peso e de peso relativo dos órgãos internos (fígado, rins, coração e baço) dos animais submetidos aos diferentes tratamentos não diferiram estatisticamente entre si (Tabela 1). Tais resultados são concordantes com os encontrados em diversos trabalhos. BAPTISTA et al. (2001; 2002 e 2003) observaram que ratos albinos da linhagem Wistar ao receberem dietas contaminadas com aflatoxinas não apresentaram diferenças significativas para esses parâmetros, quando comparados com animais que receberam dietas livres de aflatoxinas. Justificaram suas observações com base no trabalho de BARNES e BUTLER (1964), os quais relataram que ratos são animais tolerantes à toxicidade promovida pelas aflatoxinas. As médias de conversão alimentar foram semelhantes entre si para os diferentes tratamentos, exceto para os animais que receberam o tratamento 3 (médias de conversão alimentar menores do que os animais submetidos ao tratamento 4). Tal fato indica que os animais que receberam dietas contaminadas por aflatoxinas na presença de selenito de sódio evidenciaram maior habilidade para aproveitar os alimentos do que aqueles que receberam dietas contaminadas por aflatoxinas na presença de 
selênio (proveniente de leveduras). Esses resultados estão de acordo com SHI et al. (1995), que verificaram maior eficiência do selenito de sódio para diminuir a citotoxicidade induzida pelas aflatoxinas do que o selênio orgânico proveniente de leveduras.

\section{TABELA 1 - PARÂMETROS DE AVALIAÇÃO DO APROVEITAMENTO DOS ALIMENTOS E PESO RELATIVO DE ÓRGÃOS INTERNOS}

\begin{tabular}{|c|c|c|c|c|c|c|c|}
\hline \multirow[b]{2}{*}{ Tratamentos } & \multirow{2}{*}{$\begin{array}{l}\text { Consumo } \\
\text { médio } \\
\text { (g/dia) }\end{array}$} & \multirow{2}{*}{$\begin{array}{c}\text { Ganho de } \\
\text { peso } \\
\text { médio } \\
\text { (g/dia) }\end{array}$} & \multirow{2}{*}{$\begin{array}{l}\text { Conversão } \\
\text { alimentar } \\
(\%)\end{array}$} & \multicolumn{4}{|c|}{ Peso relativo por 100 gramas de peso vivo } \\
\hline & & & & $\begin{array}{l}\text { Fígado } \\
\text { (g) }\end{array}$ & $\begin{array}{l}\text { Rins } \\
\text { (g) }\end{array}$ & $\begin{array}{l}\text { Coração } \\
\text { (g) }\end{array}$ & $\begin{array}{l}\text { Baço } \\
(\mathrm{g})\end{array}$ \\
\hline T1 & $5,00 \mathrm{a}$ & $14,52 \mathrm{a}$ & $2,95 \mathrm{ab}$ & $3,62 \mathrm{a}$ & $0,95 a$ & $0,49 a$ & $0,38 a$ \\
\hline $\mathrm{T} 2$ & $4,90 \mathrm{a}$ & $12,60 \mathrm{a}$ & $2,62 a b$ & $4,03 \mathrm{a}$ & $1,02 \mathrm{a}$ & $0,42 \mathrm{a}$ & $0,47 \mathrm{a}$ \\
\hline T3 & $4,90 \mathrm{a}$ & $12,38 a$ & $2,53 \mathrm{~b}$ & $4,12 \mathrm{a}$ & $1,00 \mathrm{a}$ & $0,40 \mathrm{a}$ & $0,46 \mathrm{a}$ \\
\hline T4 & $4,67 \mathrm{a}$ & $18,65 \mathrm{a}$ & $3,99 a$ & $3,90 \mathrm{a}$ & $0,98 \mathrm{a}$ & $0,47 \mathrm{a}$ & $0,44 a$ \\
\hline T5 & $5,60 a$ & $14,80 \mathrm{a}$ & $2,66 \mathrm{ab}$ & $3,87 \mathrm{a}$ & $0,96 \mathrm{a}$ & $0,46 \mathrm{a}$ & $0,38 a$ \\
\hline T6 & $4,62 \mathrm{a}$ & $14,40 \mathrm{a}$ & $3,11 a b$ & $3,64 \mathrm{a}$ & $0,91 \mathrm{a}$ & $0,45 \mathrm{a}$ & $0,40 \mathrm{a}$ \\
\hline
\end{tabular}

Médias seguidas por letras distintas, na mesma coluna, diferem estatisticamente entre si ao nível de significância de 0,05 de probabilidade pelo teste de Tukey.

\subsection{DIAGNÓSTICO HISTOPATOLÓGICO}

Os resultados obtidos demonstraram que os tecidos hepáticos dos animais que receberam dietas livres de aflatoxinas não apresentaram sinais de intoxicação (Figura 1). Os animais que receberam dietas somente com aflatoxinas (grupo 2) não tiveram seus tecidos hepáticos muito alterados, apresentando poucos danos celulares e algumas vacuolizações que poderiam ser edema ou alteração gordurosa. Contudo, as modificações indicaram que as alterações encontravam-se em estado inicial (Figura 2) e podiam se agravar caso a exposição à toxina fosse continuada, ou serem revertidas ao quadro clínico normal com a suspensão do tratamento. Esses resultados corroboram parcialmente os encontrados por HEATCOTHE e HIBBERT, 1978 e ELLIS, SMITH e SIMPSON, 1991 que relataram sinais evidentes e pronunciados de hepatotoxicidade em animais que ingeriram dietas contaminadas por aflatoxinas. Essas diferenças podem ser atribuídas ao modo como foi conduzida esta pesquisa, uma vez que inicialmente foi ministrada dose menor de aflatoxinas $\left(255 \mu \mathrm{g} \mathrm{kg}^{-1}\right)$ com aumento nessa contaminação para aproximadamente $600 \mu \mathrm{g} \mathrm{kg}^{-1}$. Os resultados indicam que o fato de se fornecer dose inicial baixa parece ter induzido tolerância às maiores doses de aflatoxinas e com isso os danos celulares apresentados após o período experimental não foram muito pronunciados. Tal fato pode ser explicado pelas afirmações de ROBBINS, ANGELL e KUMAR (1986), os quais relataram que quando os distúrbios ocorridos em uma célula não são muito pronunciados, essa tende a se adaptar e com isso pode superar a condição desfavorável a qual foi submetida.

Os animais que receberam dietas com AF (M) mais $\mathrm{Na}_{2} \mathrm{SeO}_{4}$ e AF (M) mais SeL apresentaram tecidos hepáticos muito bem preservados, com menos vacuolizações do que os que receberam dietas com $\mathrm{AF}(\mathrm{M})$. Tal fato indica que o selênio promoveu efeito protetor razoável contra aflatoxinas (Figuras 3 e 4). Esses resultados reforçam os relatos dos trabalhos conduzidos por LEl et al.(1990); NYANDIEKA, WAKHIS e KILONZO (1990) e SHI et al. (1994 e 1995), os quais descreveram que o selênio parecia apresentar propriedades protetoras contra as aflatoxinas. Além disso, atribuíram tal capacidade inibitória aos danos promovidos por aflatoxinas ao potencial antioxidante do selênio. 
FIGURA 1 - FOTOMICROGRAFIA DO TECIDO HEPÁTICO DE RATOS ALIMENTADOS COM DIETA LIVRE DE AFLATOXINAS*

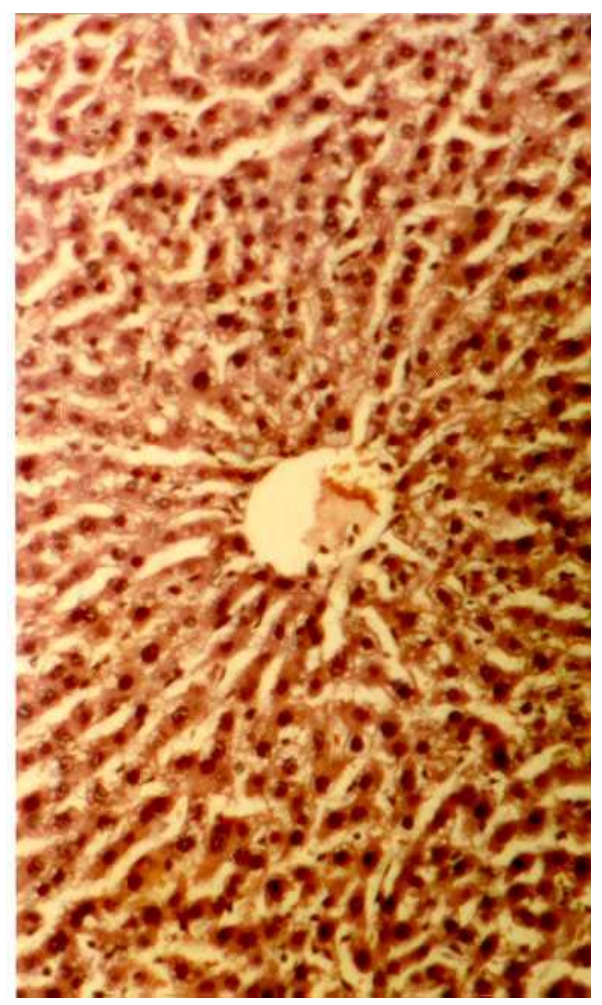

FIGURA 3 - FOTOMICROGRAFIA DO TECIDO HEPÁTICO DE RATOS ALIMENTADOS COM DIETA CONTAMINADA POR AFLATOXINAS + SELENITO DE SÓDIO*

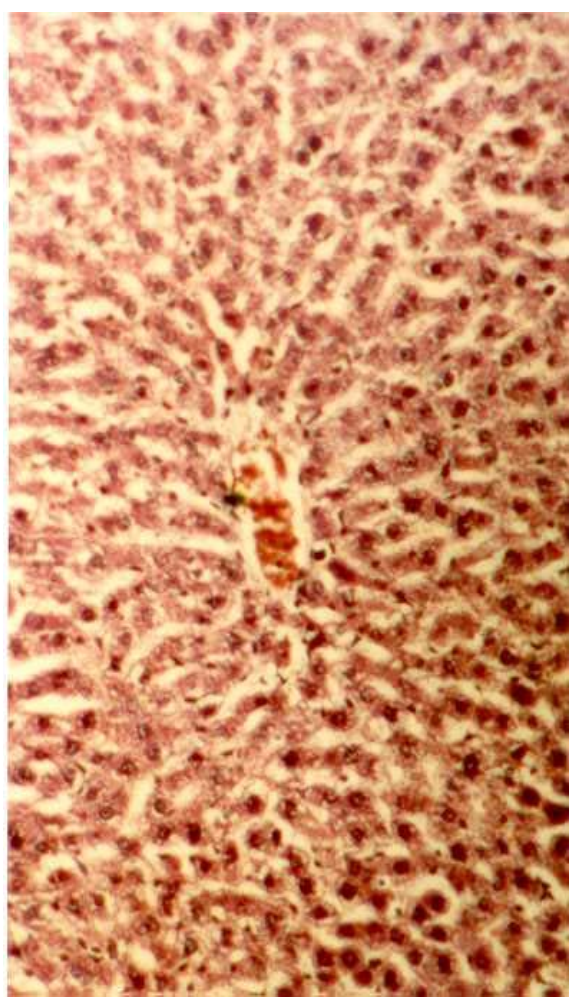

FIGURA 2 - FOTOMICROGRAFIA DO TECIDO HEPÁTICO DE RATOS ALIMENTADOS COM DIETA CONTAMINADA POR AFLATOXINAS*

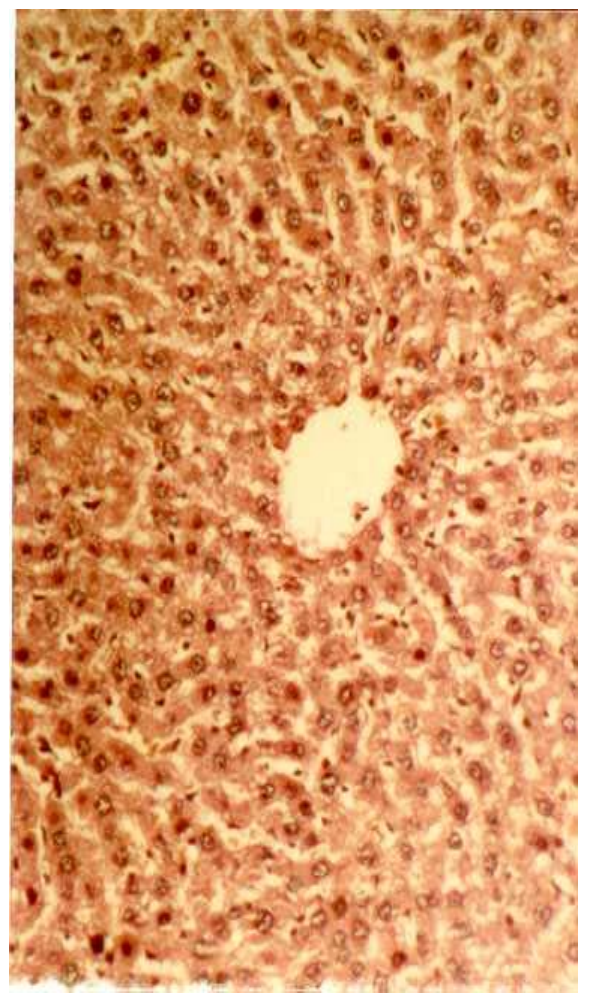

FIGURA 4 - FOTOMICROGRAFIA DO TECIDO HEPÁTICO DE RATOS ALIMENTADOS COM DIETA CONTAMINADA POR AFLATOXINAS + SELÊNIO PROVENIENTE DE LEVEDURAS*

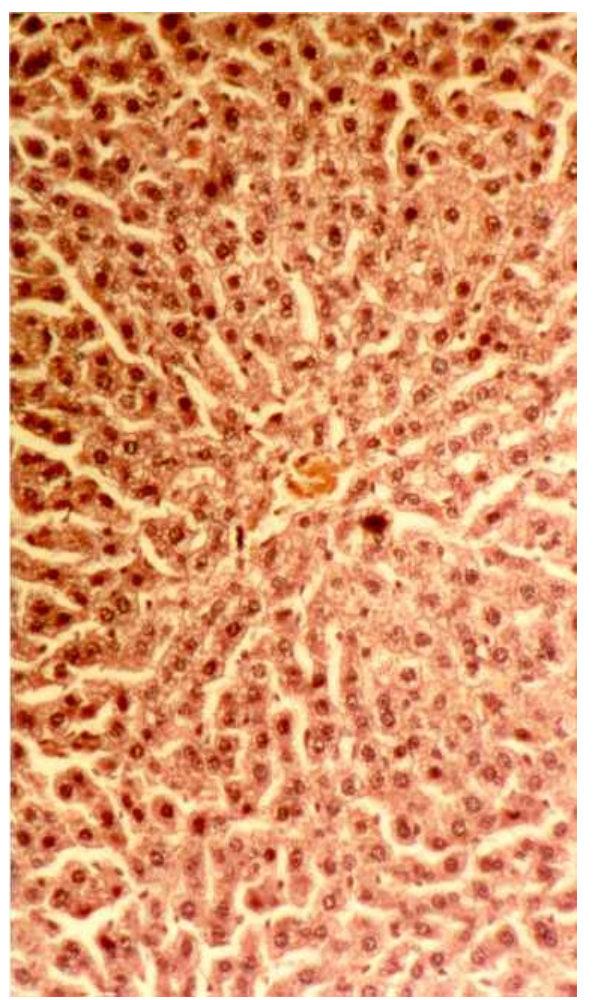

*Aumento de $200 \mathrm{X}$. 
Os ratos submetidos aos tratamentos com dieta-padrão suplementada com $\mathrm{Na}_{2} \mathrm{SeO}_{4}$ ou com SeL puros apresentaram alterações hepáticas, com vacuolizações e poucas figuras de necrose de núcleo, indicando que as doses ministradas puras podem induzir hepatotoxidez (Figuras 5 e 6). Esses resultados podem ser explicados por relatos de outros autores, que afirmaram que níveis inadequados de selênio podem ser tóxicos e promover alterações celulares (FRANCO, 1992; SCHRAUZER, 2000).

\section{FIGURA 5 - FOTOMICROGRAFIA DO TECIDO HEPÁTICO DE RATOS ALIMENTADOS COM DIETA LIVRE DE AFLATOXINAS + SELENITO DE SÓDIO*}

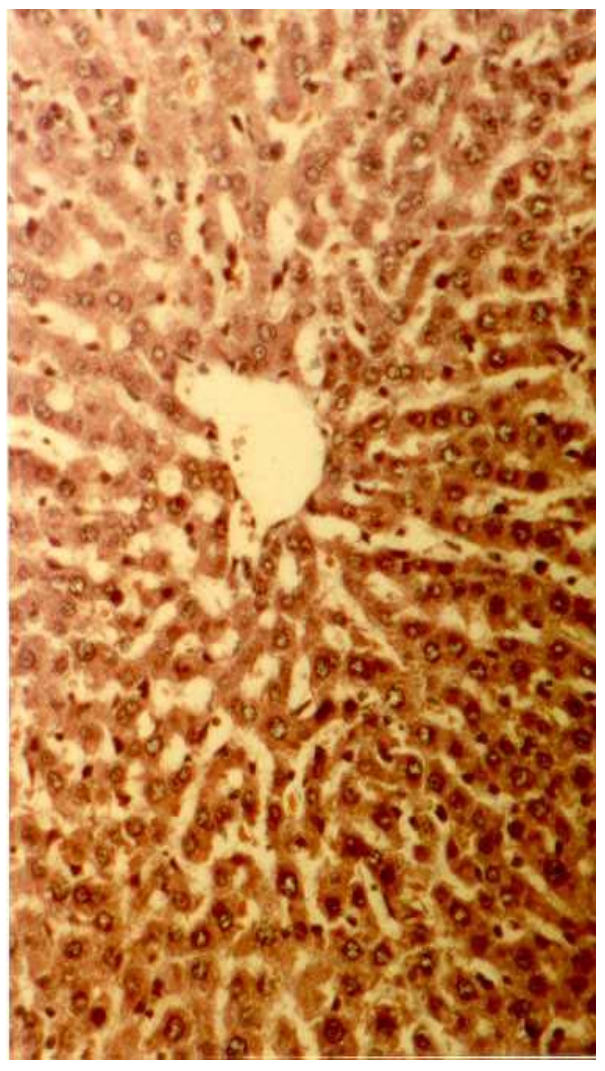

\section{FIGURA 6 - FOTOMICROGRAFIA DO TECIDO HEPÁTICO DE RATOS ALIMENTADOS COM DIETA LIVRE DE AFLATOXINAS + SELÊNIO PROVENIENTE DE LEVEDURAS*}

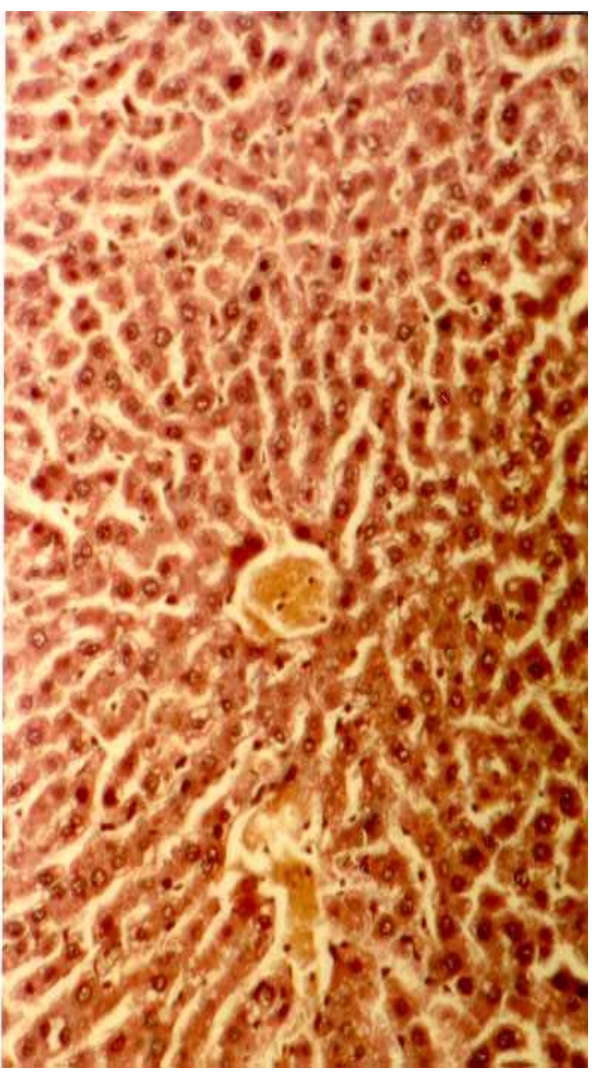

* Aumento de $200 \mathrm{X}$.

\section{CONCLUSÃO}

Os animais que receberam suplementação alimentar com selenito de sódio em dieta contaminada com aflatoxinas apresentaram maior conversão alimentar do que aqueles que recebem suplementação com selênio proveniente de leveduras sob as mesmas condições.

Embora o selenito de sódio e o selênio proveniente de leveduras apresentem potencial para reduzir os danos induzidos por aflatoxinas, o emprego de altas doses desse antioxidante em dietas livres de aflatoxinas pode induzir à hepatotoxicidade em animais. Desta forma, a suplementação desse mineral em doses acima do normal deve ser recomendada somente para os casos em que as dietas estiverem contaminadas com aflatoxinas. 


\section{ABSTRACT \\ CAPACITY OF TWO SOURCES OF SELENIUM TO REDUCE HEPATOTOCIXITY CAUSED BY AFLATOXINS INGESTION}

Eighteen wistar rats were used to evaluate the effect of sodium selenate $\left(\mathrm{Na}_{2} \mathrm{SeO}_{4}\right)$ and of selenium originating from yeasts (SeL) on the damages induced by aflatoxins. The animals were distributed in six groups, of three repetition each: group $1=$ control; group $2=480 \mu$ aflatoxins $\mathrm{kg}^{-1}(\mathrm{AF})$; group $3=0.5 \mathrm{mg}$ of $\mathrm{Na}_{2} \mathrm{SeO}_{4}$ per $\mathrm{kg}$ of diet; group $4=0.5 \mathrm{mg}$ of SeL per $\mathrm{kg}$ of diet; group $5=\mathrm{AF}+0.5 \mathrm{mg}$ of $\mathrm{Na}_{2} \mathrm{SeO}_{4}$ per $\mathrm{kg}$ of diet and group $6=\mathrm{AF}+0.5 \mathrm{mg}$ of SeL per $\mathrm{kg}$ of diet. After 28 days of essay, the animals were slaughtered for the histo-pathological study. The animals of the group AF showed few hepatotoxicity signs. In the groups AF plus $\mathrm{Na}_{2} \mathrm{SeO}_{4}$ and $\mathrm{AF}$ plus SeL, the animals presented livers very well preserved. The animals that received $\mathrm{Na}_{2} \mathrm{SeO}_{4}$ or SeL showed some alterations. In conclusion, the sodium selenate and SeL showed potential to reduce the damages caused by aflatoxins.

KEY-WORDS: MICOTOXINS; ANTIOXIDANT; YEAST; LIVER; GLUTATHIONE PEROXIDASE.

\section{REFERÊNCIAS}

1 BAPTISTA, A. S.; HORII, J.; CALORI-DOMINGUES, M. A.; GLÓRIA, E. M.; SALGADO, J. M., VIZIOLI, M. R. Saccharomyces cerevisiae in the reduction of the aflatoxins effects. European Journal of Pharmaceutical Sciences, v.13, p.548-548, 2001.

2 BAPTISTA, A. S.; HORII, J.; CALORI-DOMINGUES, M. A.; GLÓRIA, E. M.; SALGADO, J. M.; VIZIOLI, M. R. Thermolysed and active yeast to reduce to toxicity of aflatoxin. Scientia Agricola, v.59, n.2, p.257-260, 2002.

3 BAPTISTA, A. S.; ABDALLA, A. L.; PIRES, D. S.; ZAMPRONIO, A. C.; GLÓRIA, E. M.; CALORI-DOMINGUES, M. A.; HORII, J.; VIZIOLI, M. R. Diferentes linhagens de Sacchomyces cerevisiae no controle de aflatoxicoses In: CONGRESSO BRASILEIRO DE MICROBIOLOGIA, 22, 2003, Florianópolis. Resumo... Florianópolis: Sociedade Brasileira de Microbiologia, 2003. p. 102

4 BARNES, J. M.; BUTLER, W. H. Carcinogenisis activity of aflatoxin in rats. Nature, v.202, p.1016-1016, 1964.

5 ELLIS, W. O.; SMITH, J. P.; SIMPSON, B. K. Aflatoxins in food: occurrence, biosynthesis, effects on organisms, detection, and methods of control. Critical Reviews in Food Science and Nutrition, v.30, n.4, p.403-439, 1991.

6 FRANCO, G. Tabela de composição química dos alimentos. 9. ed. São Paulo: Ateneu, 1992

7 HAMILTON, P. B. Determining safe concentrations of mycotoxins. Journal of Food Protection, v.47, n.7, p.570-575, 1984.

8 HEATHCOTE, J. G.; HIBBERT, J. R. Aflatoxins: chemical and biological aspects. Amsterdam: Elsevier, 1978. 212 p.

9 HERZBERG, P. A. How SAS works: a comprehensive introduction to the SAS system. Ottawa: Captus Press, 1990. $193 \mathrm{p}$.

10 KUBENA, L. E.; HARVEY, R. B.; PHILLIPS, T. D.; CORRIER, D. E.; HUFF, W. E. Diminution of aflatoxicosis in growing chickens by the dietary addition of a hydrated sodium calcium aluminosilicate. Poultry Science, v.69, p.727-735, 1990.

11 LEI, D. N.; WANG, L. Q.; RUEBNER, B. H.; HSIEH, D. P.; WU, B. F.; ZHU, C. R.; DU, M. J. Effect of selenium on aflatoxin hepatocarcinogenesis in the rat. Biomedical and Environmental Sciences, v.3, p.65-80, 1990.

12 NYANDIEKA, H. S.; WAKHIS, J.; KILONZO, M. M. Association of reduction of AFB1-induced liver-tumors by antioxidants with increased activity of microsomal-enzymes. Indian Journal of Medical Research, Section B-Biomedical Research other than Infectious Diseases, v.92, p.332-336, 1990.

13 PACHECO, A. C. Compêndio básico de técnicas histológicas. Botucatu: Universidade Estadual Paulista Júlio de Mesquita Filho, 1981.

14 REEVES, P. G.; NIELSEN, F. H.; FAHEY, G. C. Ain-93 purified diets for laboratory rodents - final report of the American Institute of Nutrition ad hoc Writing Committee on the reformulation of the ain-76a rodent diet. Journal of Nutrition, v.23, n.11, p.1939-1951, 1993. 
ROBBINS, S. L.; ANGELL, M.; KUMAR, V. Patologia básica. São Paulo: Ateneu, 1986. p.3-31.

16 ROTRUCK, J. T.; POPE, A. L.; GANTHER, H. E.; SWANSON, A. B.; HAFEMAN, D. G.; HOEKSTRA, W. G. Selenium - biochemical role as a component of glutathione peroxidase. Science, v.179, n.4073, p.588-590, 1973.

17 SCHRAUZER, G. N. Selenomethionine: a review of its nutritional significance, metabolism and toxicity. Journal of Nutrition, v.130, n.7, p.1653-1656, 2000.

18 SHI, C. Y.; CHUA, S. C.; LE, H. P.; ONG, C. N. Inhibition of aflatoxin B-1-DNA binding and adduct formation by selenium in rats. Cancer Letters, v.82, n.2, p.203-208, 1994.

19 SHI, C. Y.; HEW, Y. C.; ONG, C. N. Inhibition of aflatoxin b1-induced cell injury by selenium - an in-vitro study. Human \& Experimental Toxicology, v.14, n.1, p.55-60, 1995.

20 SOARES, L. M. V; RODRIGUES-AMAYA, D. B. Survey of aflatoxins, ochratoxin A, zearalenone and sterigmatocystin in some Brazilian food by using multi-toxin thin-layer chromatografic method. Journal of Official Analytical Chemists, v.72, p.22-6, 1989.

\section{AGRADECIMENTOS}

Ao Sr. Lécio Aparecido Castilho, Técnico do Laboratório de Nutrição Animal (LANA/CENA/USP), pelo auxílio na condução dos bioensaios; À Ivani V. Zambello, do Laboratório de Micotoxinas (LAN/ESALQ/USP), que ajudou a executar as análises de micotoxinas. 